\title{
Oldhamianoside II inhibits prostate cancer progression via regulation of EMT and the Wnt/ $\beta$-catenin signaling pathway
}

\author{
KAIZHI LI ${ }^{1 *}$, XUEMEI ZHAN ${ }^{2 *}$, JINGYONG SUN $^{3}$, TIANFENG WANG ${ }^{4}$, HONGYAN DONG $^{2}$, \\ FANBO JING ${ }^{5}$, DONGMEI LI ${ }^{5}$, YU CAO ${ }^{5}$, YALI LIU ${ }^{5}$, LIN WANG ${ }^{6}$ and SHENGNAN LI ${ }^{7}$ \\ ${ }^{1}$ Department of Pathology, Affiliated Hospital of Jining Medical University, Jining, Shandong 272029; \\ ${ }^{2}$ Department of Pathology, Linyi People's Hospital, Linyi, Shandong 276000; ${ }^{3}$ Department of Pharmacology, \\ Shandong Academy of Medical Sciences, Jinan, Shandong 250062; ${ }^{4}$ Department of Hepatobiliary Surgery, \\ Linyi People's Hospital, Linyi, Shandong 276000; ${ }^{5}$ Department of Pharmacy, The Affiliated Hospital of Qingdao University, \\ Qingdao, Shandong 266000; ${ }^{6}$ Shandong Medical Biotechnological Center, Key Laboratory for Rare and Uncommon Diseases \\ of Shandong Province, Shandong Academy of Medical Sciences, Jinan, Shandong 250062; 7 Department of Pharmacy, \\ The Affiliated Cardiovascular Hospital of Qingdao University, Qingdao, Shandong 266000, P.R. China
}

Received December 11, 2015; Accepted January 25, 2018

DOI: $10.3892 / \mathrm{ol} .2018 .8450$

\begin{abstract}
Oldhamianoside II is a novel triterpenoidsaponin that can be isolated from the roots of Gypsophila oldhamiana. In vitro and in vivo experiments have revealed that it inhibits tumor growth and metastasis in various types of tumor; however, the exact mechanism remains to be fully elucidated. In the present study, oldhamianoside II treatment in prostate cancer cells exerted substantial anticancer activity, including decreased cell proliferation and invasion. Mechanistically, oldhamianoside II was found to reverse the epithelial-mesenchymal transition (EMT), as demonstrated by its induction of E-cadherin and suppression of vimentin and N-cadherin at the mRNA and protein levels. Furthermore, oldhamianoside II treatment upregulated Wnt antagonist expression and promoted the proteasome-mediated degradation of $\beta$-catenin to inhibit the activity of $\beta$-catenin signaling. In summary, the present study revealed that oldhamianoside II exerts its antitumor effects via the regulation of EMT and $\beta$-catenin
\end{abstract}

Correspondence to: Dr Shengnan Li, Department of Pharmacy, The Affiliated Cardiovascular Hospital of Qingdao University, 5 Zhiquan Road, Qingdao, Shandong 266000, P.R. China

E-mail: yysghyy@163.com

Dr Lin Wang, Shandong Medical Biotechnological Center, Key Laboratory for Rare and Uncommon Diseases of Shandong Province, Shandong Academy of Medical Sciences, 18877 Jingshi Road, Jinan, Shandong 250062, P.R. China

E-mail: wanglin.83@163.com

${ }^{*}$ Contributed equally

Key words: prostate cancer, oldhamianoside II, $\beta$-catenin, epithelial-mesenchymal transition function, and further supports its potential for use in clinical treatment.

\section{Introduction}

Prostate Cancer (PCa) is the second most frequently diagnosed cancer among men and the fifth leading cause of cancer mortalities worldwide (1). Mortality rates for PCa have been decreasing in the majority of more developed countries, but continue to rise in certain Asian countries, particularly in China (1). The primary diagnostic tools used to obtain evidence of PCa include digital rectal examination, serum concentration of PSA and transrectal ultrasound guided biopsies (2). Diagnosis depends on the presence of adenocarcinoma in operative specimens and prostate biopsy cores (2). The factors driving the increase of incidence in prostate cancer are not entirely understood; however, they may include gradual implementation of prostate-specific antigen screening and improved biopsy techniques, or the effect of an increasingly westernized lifestyle, in particular dietary habits (1). Despite recent hormonal therapies exhibiting a survival advantage during treatment with inhibitors, including abiraterone acetate (17 $\alpha$-hydroxylase inhibitor) and enzalutamide (androgen receptor inhibitor), the median duration of response is $<1$ year (2). Furthermore, the treatment options for recurrent and metastatic PCa are limited and predominantly palliative (2).

Epithelial-mesenchymal transition (EMT) is an early embryonic development program, in which cells convert from the epithelial to the mesenchymal state (3). During this process, the epithelial cells acquire mesenchymal cell morphology through the suppressing the epithelial markers and inducing mesenchymal markers (4). It has been shown to have a critical role during prostate cancer progression and metastasis (5). Another important signaling pathway is Wingless (WNT)/ $\beta$-catenin (6) and activation of Wnt $/ \beta$-catenin signaling induces motility, invasiveness, cell-fate decisions and the maintenance of self-renewal potential (7). Recent studies 
indicate that, remarkably, Wnt/ $\beta$-catenin signaling is essential for the biological process of bone metastasis in various types of cancers, particularly in $\mathrm{PCa}(8)$.

Oldhamianoside II, atriterpenoidsaponin, may be isolated from Gypsophila oldhamiana, a plant that is rich in saponins (9). A previous study demonstrated that oldhamianoside II was able to effectively inhibit the proliferation and migration of tumor cells, as well as tumor angiogenesis (10). These results indicated the feasibility of oldhamianoside II for further clinical application. A previous study demonstrated that oldhamianoside II exerted antitumor activity via inhibition of $\mathrm{C}-\mathrm{X}-\mathrm{C}$ motif chemokine receptor 4 , matrix metalloproteinase 2, phosphoinositide 3-kinase/protein kinase B and extracellular signal-related kinase/mitogen activated protein kinase (10); however, the specific mechanisms underlying the antitumor effects of oldhamianoside II in PCa remain unclear. The present study aimed to further elucidate these mechanisms, and revealed that oldhamianoside II inhibited the proliferation and invasion of PCa cells by regulating the process of epithelial-mesenchymal transition (EMT) and the activity of the Wnt/ $\beta$-catenin signaling pathway.

\section{Materials and methods}

Cell culture and treatment. VCaP and PC3 human PCa cell lines were purchased from American Type Culture Collection (Manassas, VA, USA) and maintained in Dulbecco's modified Eagle's medium (HyClone; GE Healthcare, Chicago, IL, USA) or RPMI-1640 (HyClone; GE Healthcare) supplemented with $10 \%$ fetal bovine serum (Invitrogen; Thermo Fisher Scientific, Inc., Waltham, MA, USA). For the experiments, cells were cultured at $37^{\circ} \mathrm{C}$ and stimulated with oldhamianoside II, which was extracted and isolated as previously described (9). Cycloheximide (50 $\mu \mathrm{g} / \mathrm{ml}$, CHX; Sigma-Aldrich; Merck KGaA, Darmstadt, Germany) or MG132 (30 $\mu \mathrm{M}$, Sigma-Aldrich; Merck KGaA) were applied to stimulate PCa cells for $6 \mathrm{~h}$ before oldhamianoside II treatment.

MTT assay. An MTT assay was performed to detect to the cell viability as previously described (11). Briefly, $1 \times 10^{4}$ cells per well in $200 \mu \mathrm{l}$ medium were seeded into a 96 -well plate and then subjected to the indicated treatments for 24-72 h at $37^{\circ} \mathrm{C}$. For detection, $20 \mu \mathrm{l} \mathrm{MTT}$ was added to each well and incubated for $4 \mathrm{~h}$. Subsequently, the cultured medium was discarded and $150 \mu \mathrm{l}$ dimethyl sulfoxide was added. The absorbance was then determined at $490 \mathrm{~nm}$, and all experiments were performed in triplicate.

Migration assay. For the migration assay, $1 \times 10^{4}$ cells per well in serum-free medium were plated into 24-well Transwell chambers with polycarbonate filter inserts (pore size, 8.0-10.0 $\mu \mathrm{m}$; Corning Incorporated, Corning, NY, USA). The lower chambers contained normal growth medium with $10 \%$ fetal bovine serum. Following incubation at $37^{\circ} \mathrm{C}$ for $24 \mathrm{~h}$, the cells in the upper compartment were removed and the migrated cells were fixed and stained using the crystal violet method. Cells were quantified in five randomly selected microscopic fields using light microscope (magnification, x200) and all experiments were performed in triplicate.
$R N A$ extraction and reverse transcription-quantitative polymerase chain reaction ( $R T-q P C R)$. Total RNA was extracted using an RNeasy Mini kit (Qiagen), and RNA (1 $\mu \mathrm{g})$ was reverse-transcribed to cDNA using a SuperScript II cDNA synthesis kit (Invitrogen). Subsequently, $1 \mu \mathrm{g}$ of cDNA product was used for amplification with the PCR amplification kit (Takara) as previously described (12). The details of the primers for each human gene were as follows: E-cadherin forward, 5'-TCATGAGTGTCCCCCGGTAT-3', and reverse, 5'-TCTTGAAGCGATTGCCCCAT-3'; N-cadherin forward, 5'-CGCCATCCGCTCCACTT-3', and reverse, 5'-GCTGAT GACAAATAGCGGGC-3'; vimentin forward, 5'-GGACCA GCTAACCAACGACA-3', and reverse, 5'-AAGGTCAAG ACGTGCCAGAG-3'; cyclin D1 (CCND1) forward, 5'-CAC ACGGACTACAGGGGAGT-3', and reverse, 5'-GATGGT TTCCACTTCGCAGC-3'; c-JUN forward, 5'-GTGCCGAAA AAGGAAGCTGG-3', and reverse, 5'-CTGCGTTAGCAT GAGTTGGC-3'; axin 2 (AXIN2) forward, 5'-AAACGCAAT GGGAAAGGCAC-3', and reverse, 5'-TGTGCTTTGGGC ACTATGGG-3'; Dickkopf-1 (DKK1) forward, 5'-AAGTGT GGTGGCTTCCAAGG-3', and reverse, 5'-CCGGCCACA TGAGTAAGAGG-3'; protein phosphatase 2 catalytic subunit $\beta$ (PPP2CB) forward, 5'-ACCATGCCAATGGTCTCACA-3', and reverse, 5'-AACATGAGGCTCACCACGAC-3'; secreted frizzled-related protein 5 (SFRP5) forward, 5'-CACTCGGAT ACGCAGGTCTT-3', and reverse, 5'-CACTCGGATACGCAG GTCTT-3'; and GAPDH (internal loading control) forward, 5'-AATGGGCAGCCGTTAGGAAA-3', and reverse, 5'-GCG CCCAATACGACCAAATC-3'. The reaction was performed at $94^{\circ} \mathrm{C}$ for $30 \mathrm{sec}, 56^{\circ} \mathrm{C}$ for $30 \mathrm{sec}$ and $72^{\circ} \mathrm{C}$ for $30 \mathrm{sec}$, for 36 cycles. GAPDH served as the loading control. Analysis of relative gene expression data using real-time quantitative PCR and the $2^{-\Delta \Delta \mathrm{Cq}}$ method (13). All reactions were performed in triplicate.

Western blot analysis. The nuclear proteins were extracted using Nuclear and Cytoplasmic Extraction Reagents kits (Thermo Fisher Scientific, Inc., Waltham, MA, USA). Western blotting was performed as previously described (14). The primary antibodies against E-cadherin (1:1,000; cat no. 3195), $\mathrm{N}$-cadherin $(1: 1,000$; cat no. 13116), $\beta$-catenin $(1: 1,000$; cat no. 8480) and Vimentin (1:1,000; cat no. 5741) were purchased from Cell Signaling Technology, Inc. (Danvers, MA, USA) and the antibodies against cyclin D1 (1:1,000; cat no. ab134175), c-Jun (1:1,000; cat no. ab32137) and Lamin A (1:1,000; cat no. ab108922) were purchased from Abcam (Cambridge, UK). For the loading control, HRP-conjugated-GAPDH antibodies (1:1,000; cat no. sc-293335; Santa Cruz Biotechnology, Inc., Dallas, TX, USA) were used. The primary antibodies were incubated at $4^{\circ} \mathrm{C}$ overnight, and the second antibody labeled with horseradish-peroxidase was subsequently incubated at $37^{\circ} \mathrm{C}$ for $1 \mathrm{~h}$. SuperSignal West Pico kit (Thermo Fisher Scientific, Inc.) was used to visualize the protein bands.

Statistical analysis. Statistical analyses of the data were performed using SPSS version 16 (SPSS, Inc., Chicago, IL, USA). Data are presented as the mean \pm standard deviation and were analyzed by one-way analysis of variance followed by Tukey's post hoc test, considering oldhamianoside II treatment and cell viability at each time point, or vehicle 


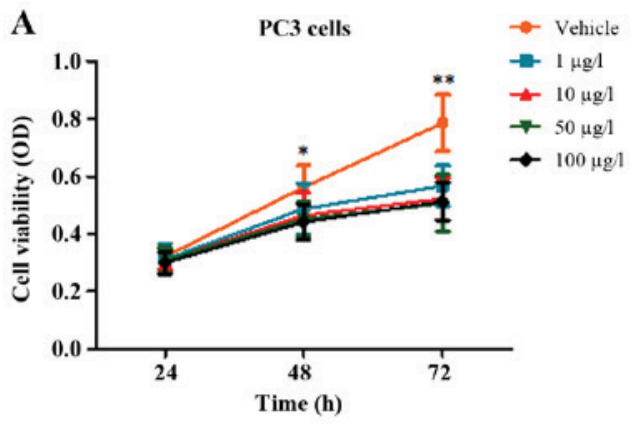

C
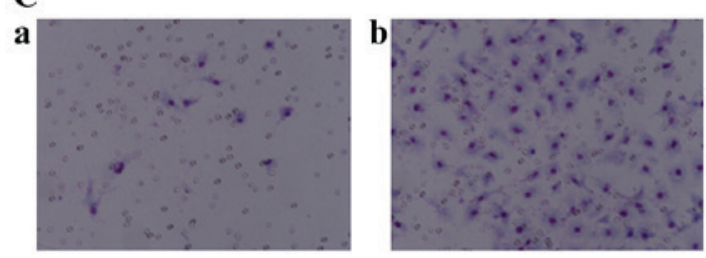

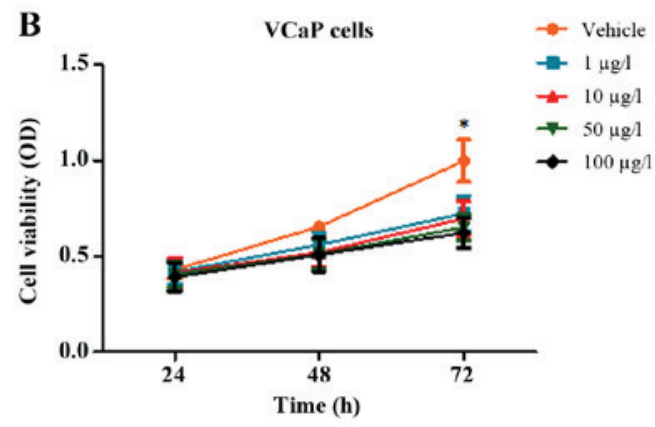

D
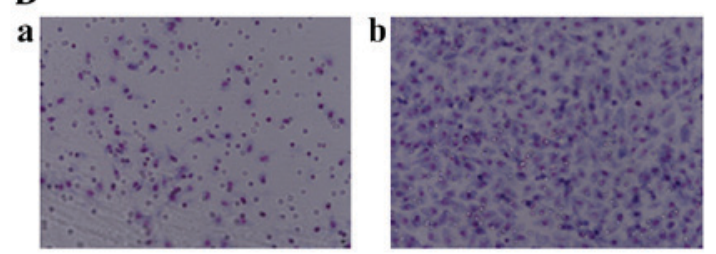

Figure 1. Effects of oldhamianoside II on prostate cancer cells. Cell viability was assessed by MTT assay at indicated timepoints (0 to 72 h) in (A) PC3 and (B) VCaP cells following oldhamianoside II treatment (1-100 $\mu \mathrm{g} / 1)$. The invasive capacity of (C) PC3 and (D) VCaP cells was assessed using invasion chambers: (a) Control (PBS) treatment group and (b) oldhamianoside II (50 $\mu \mathrm{g} / \mathrm{l})$ treatment group. The results are presented as the mean \pm standard deviation from three independent samples. ${ }^{*} \mathrm{P}<0.05$ and ${ }^{* *} \mathrm{P}<0.01$ vs. vehicle control. OD, optical density.
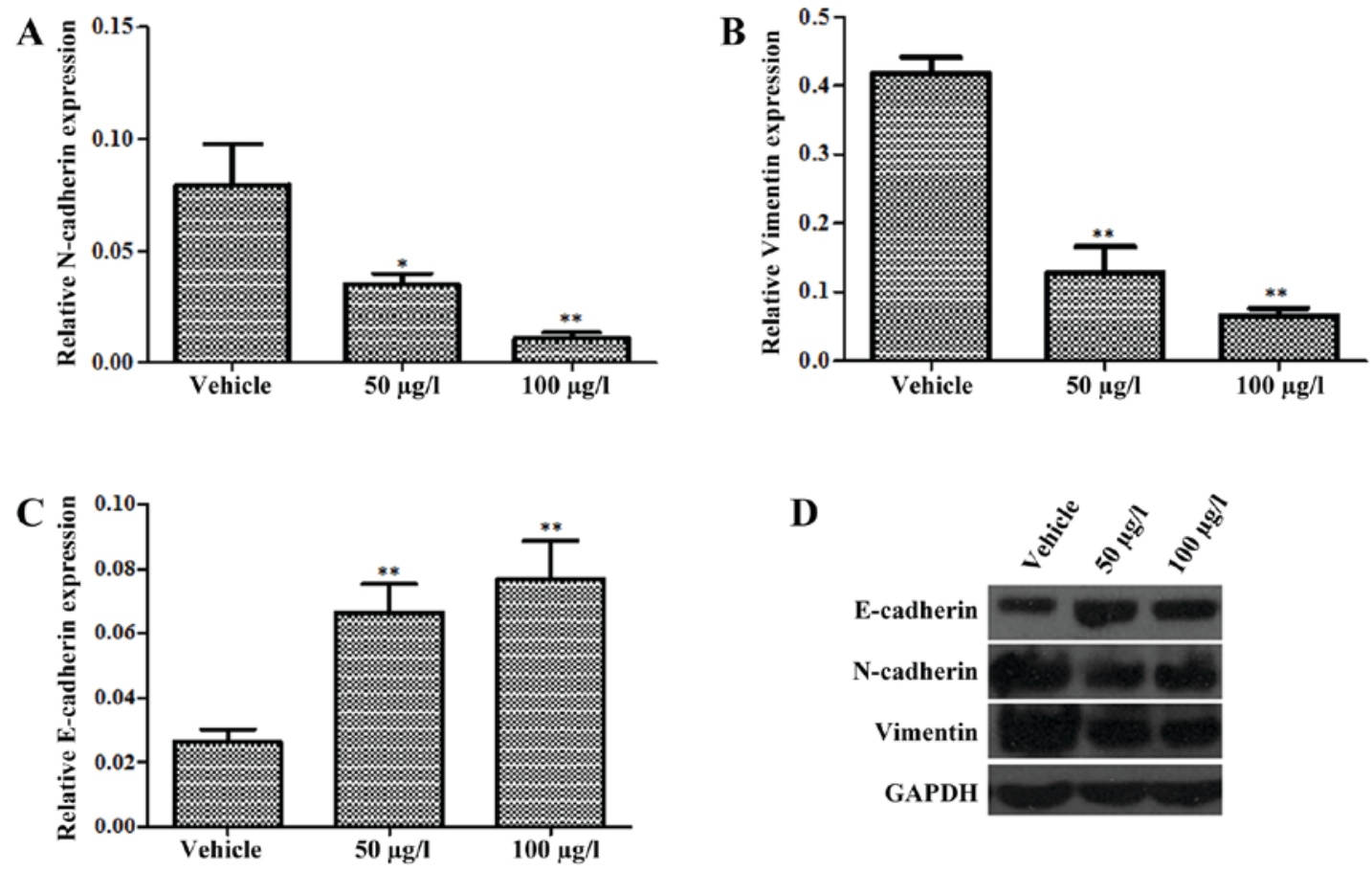

Figure 2. Oldhamianoside II inhibits epithelial-mesenchymal transition in prostate cancer cells. In oldhamianoside II-treated or untreated VCaP cells, the mRNA expression levels of the mesenchymal markers (A) Vimentin and (B) N-cadherin, and the epithelial marker (C) E-cadherin, were detected by reverse transcription-quantitative polymerase chain reaction. (D) The protein levels of Vimentin, N-cadherin, E-cadherin and $\beta$-catenin were determined by western blotting following oldhamianoside II treatment (50 and $100 \mu \mathrm{g} / \mathrm{l})$ in VCaP cells. Representative results from triplicate experiments are presented as the mean \pm standard deviation. ${ }^{*} \mathrm{P}<0.05$ and ${ }^{* *} \mathrm{P}<0.01$ vs. vehicle control.

and oldhamianoside II treatment at different concentrations. $\mathrm{P}<0.05$ was considered to indicate a statistically significant difference.

\section{Results}

Oldhamianoside II inhibits the proliferation and invasion of PCa cell lines. VCaP and PC3 human PCa cell lines were treated to detect the effects of oldhamianoside II on the biological activity of tumor cells. Consistently with a previous study (10), the MTT analysis demonstrated that oldhamianoside II treatment at various concentrations decreased the viability of PC3 and VCaP cells (Fig. 1A and B). In addition, a Transwell migration assay demonstrated that $50 \mu \mathrm{g} / 1$ oldhamianoside II significantly inhibited the migration ability of PC3 (Fig. 1C) and VCaP cells (Fig. 1D). 
A

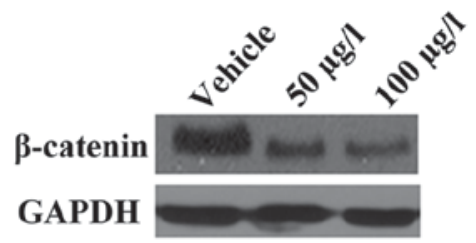

C

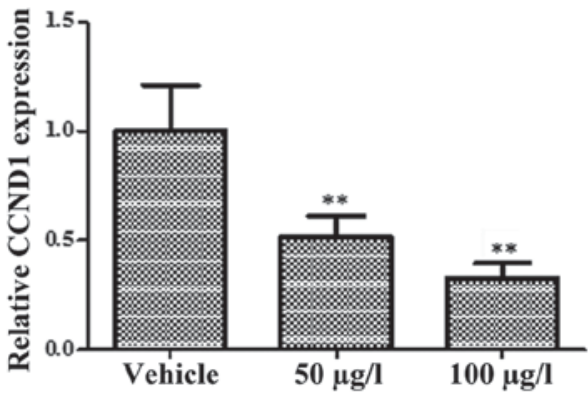

E

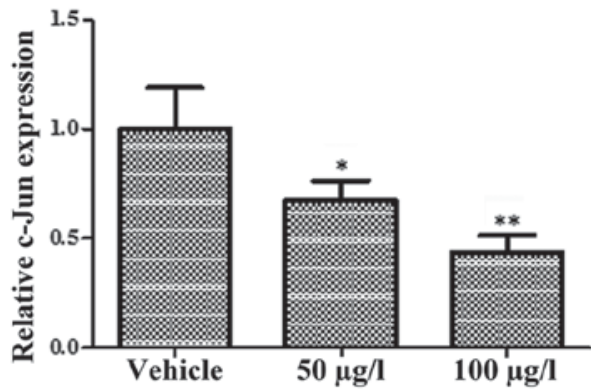

G

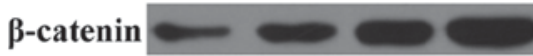

Cyclin D1

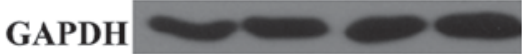

Oldhamianoside II $+\quad+\quad+\quad-$

MG132 - - + +

DMSO $++\ldots$
B

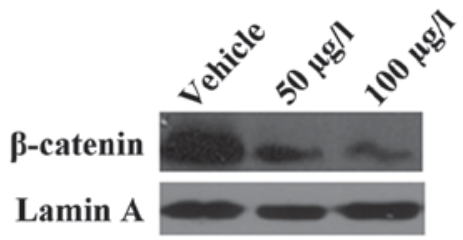

D

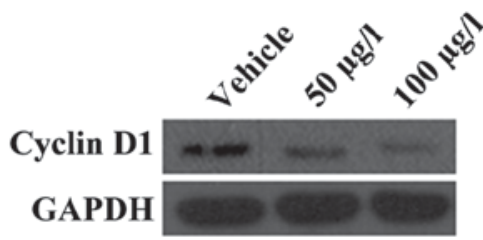

$\mathbf{F}$

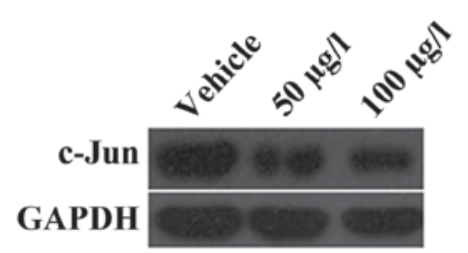

H

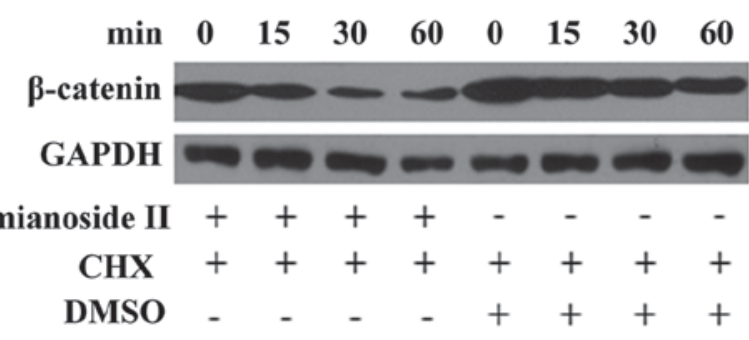

Figure 3. Oldhamianoside II functions as a negative regulator of Wnt/ $\beta$-catenin signaling and promotes $\beta$-catenin degradation in prostate cancer cells. (A) Total protein or (B) nuclear fractions from VCaP cells were analyzed by western blotting. The mRNA and protein levels of $\beta$-catenin target genes, (C and D) cyclin D1 (CCND1) and (E and F) c-Jun, in VCaP cells are shown. (G) In the presence or absence of MG132 $(30 \mu \mathrm{M})$, western blotting was performed to detect the expression of $\beta$-catenin and cyclin D1 in VCaP cells treated with or without oldhamianoside II $(100 \mu \mathrm{g} / \mathrm{l})$. (H) Following stimulation with CHX $(50 \mu \mathrm{g} / \mathrm{ml})$ for $6 \mathrm{~h}, \mathrm{VCaP}$ cells were treated with oldhamianoside II $(100 \mu \mathrm{g} / \mathrm{l})$ or its vehicle control for an additional $24 \mathrm{~h}$. Extracted total protein samples at the indicated time points were subjected to western blotting. Data are presented as the mean \pm standard deviation of three independent experiments. ${ }^{*} \mathrm{P}<0.05$ and ${ }^{* *} \mathrm{P}<0.01$ vs. vehicle control. CCND1, cyclin D1; CHX, cycloheximide.

Oldhamianoside II inhibits EMT in VCaP cells. EMT is associated with the metastasis and progression of $\mathrm{PCa}(15)$. As presented in Fig. 2A, treatment with 50 and $100 \mu \mathrm{g} / 1$ oldhamianoside II in VCaP cells for $72 \mathrm{~h}$ suppressed EMT, as indicated by the suppression of $\mathrm{N}$-cadherin (Fig. 2A) and Vimentin (Fig. 2B) and the induction of E-cadherin (Fig. 2C) at the mRNA level. Furthermore, following the treatment of $\mathrm{VCaP}$ cells with oldhamianoside II at 50 and $100 \mu \mathrm{g} / \mathrm{l}$ for $48 \mathrm{~h}$, western blot analysis demonstrated that the level of E-cadherin protein was significantly elevated, whereas the levels of Vimentin and $\mathrm{N}$-cadherin were decreased significantly (Fig. 2D).

Oldhamianoside II inhibits the expression and activity of $\beta$-catenin in PCa cells. Based on the aforementioned results, the present study investigated whether oldhamianoside II was able to regulate the activity of the $\mathrm{Wnt} / \beta$-catenin signaling pathway in $\mathrm{PCa}$ cells. Therefore, $\mathrm{VCaP}$ cells were treated with oldhamianoside II and the level of $\beta$-catenin was determined by western blotting. The total $\beta$-catenin protein level (Fig. 3A) and its nuclear content (Fig. 3B) were significantly suppressed in $\mathrm{VCaP}$ cells following oldhamianoside II treatment at 50 and $100 \mu \mathrm{g} / 1$. Subsequently, the expression levels of the target genes of $\beta$-catenin, CCND1 and c-JUN were also evaluated in response to oldhamianoside II treatment $(16,17)$. As shown in Fig. 3C-F, oldhamianoside II treatment induced a significant decrease in the expression levels of these target genes at the mRNA (Fig. 3C and E) and protein (Fig. 3D and F) levels. 
A
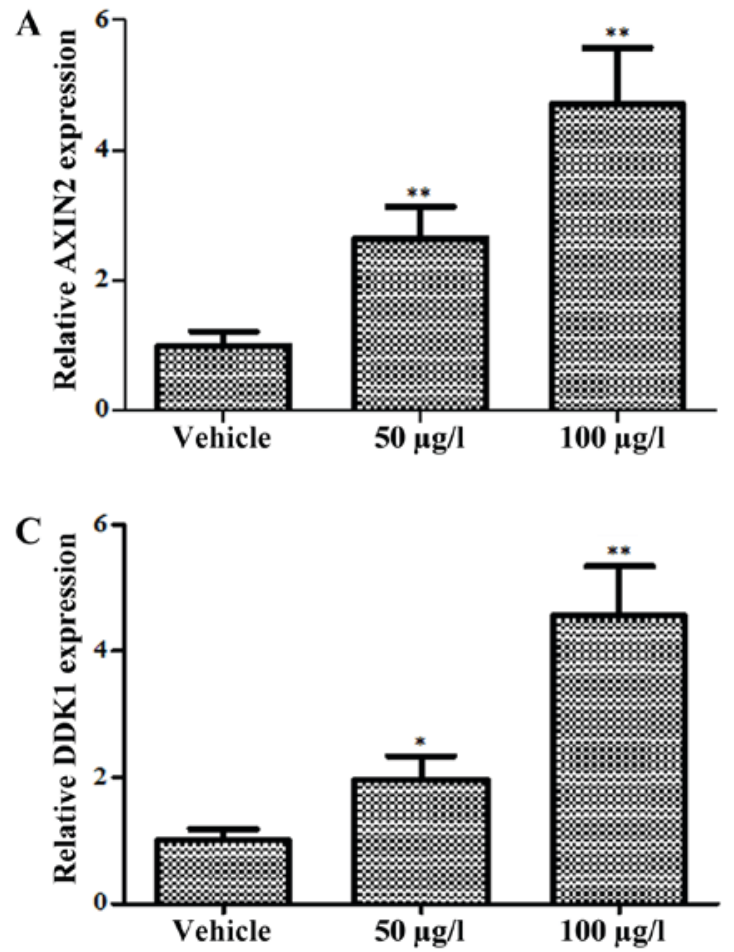

B

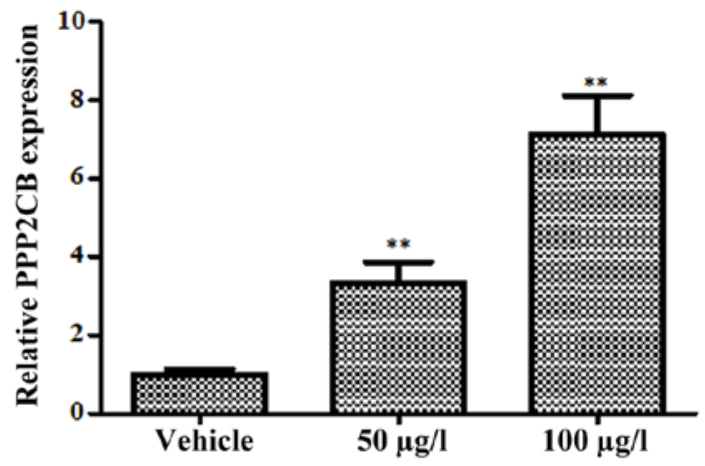

D

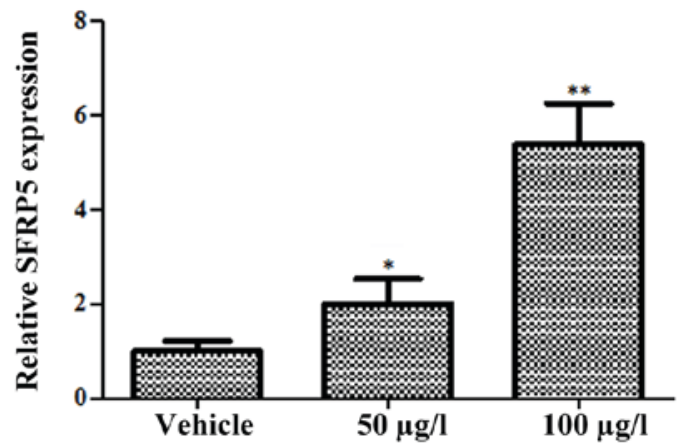

Figure 4. Oldhamianoside II transcriptionally induces Wnt antagonists. Expression levels of Wnt pathway antagonists, including (A) AXIN2, (B) PPP2CB, (C) DKK1 and (D) SFRP5, were evaluated by reverse transcription-quantitative polymerase chain reaction in VCaP cells following oldhamianoside II treatment. The mRNA expression levels of antagonists of the Wnt signaling pathway were normalized to that of GADPH. ${ }^{*}<0.05$ and ${ }^{* *} \mathrm{P}<0.01 \mathrm{vs}$. vehicle control. AXIN2, axin 2; PPP2CB, protein phosphatase 2 catalytic subunit $\beta$; DKK1, Dickkopf-1; SFRP5, secreted frizzled-related protein 5.

As $\beta$-catenin is well-validated to be targeted for ubiquitin-mediated proteolysis (18), we subsequently investigated whether oldhamianoside II was able to regulate the proteolysis of $\beta$-catenin. As presented in Fig. 3G, the effect of oldhamianoside II on $\beta$-catenin and its target cyclin D1 was blocked in the presence of the proteasome inhibitor MG132, which indicated that oldhamianoside II promoted proteasome-mediated degradation of $\beta$-catenin. Consistently, oldhamianoside II treatment resulted in a significant decrease in the half-life of $\beta$-catenin (Fig. 3H).

Oldhamianoside II promotes $\beta$-catenin degradation by inducing expression of Wnt antagonists. A previous study demonstrated that antagonists of the Wnt signaling pathway promoted $\beta$-catenin degradation by interfering with the binding of Wnt proteins to their receptors or organizing the destruction complex (19). To further confirm the regulation of oldhamianoside II on the Wnt/ $\beta$-catenin signaling pathway, the expression level of natural inhibitors towards Wnt signaling were detected following oldhamianoside II treatment. Four well-characterized antagonists (AXIN2, PPP2CB, DKK1 and SFRP5) targeting Wnt/ $\beta$-catenin signaling (20) were selected for investigation, and the results revealed that AXIN2 (Fig. 4A), PPP2CB (Fig. 4B), DKK1 (Fig. 4C) and SFRP5 (Fig. 4D) were upregulated significantly in oldhamianoside II-treated cells.

\section{Discussion}

Oldhamianoside II, a novel triterpenoidsaponin, was first chemically isolated from Gypsophila oldhamiana, commonly called 'Xia Cao' in China (9); it contains a triterpenoidaglycone and eight sugar chains. A previous study demonstrated that oldhamianoside II may inhibit tumor growth and angiogenesis, and that the migration of tumor cells was also suppressed following oldhamianoside II treatment (10). Consistent with previous evidence, the present study demonstrated that oldhamianoside II inhibited the proliferation and invasion of PCa cells.

EMT progression is characterized by cytoskeletal reorganization, followed by the suppression of cell adhesion, finally leading to enhanced cell motility. Numerous studies have validated that EMT is closely associated with tumor progression and metastasis (21). In the present study, by characterizing the expression level of epithelial and mesenchymal markers, it was demonstrated that oldhamianoside II inhibited EMT in PCa cells. Together, the aforementioned results corroborate the inhibitory role of oldhamianoside II during PCa progression.

Aberrantly activated $\mathrm{Wnt} / \beta$-catenin signaling may induce tumor formation and progression (22). $\beta$-catenin is a transcription factor that is involved in the Wnt signaling pathway and serves an important role in oncogenesis in combination with T-cell factor (Tcf) and protein kinase D1 $(23,24)$. It has previously been reported that $\beta$-catenin was dysregulated in and associated with the development of numerous types of cancer, including PCa (25), and that the shuttle between the cytoplasm and nucleus was pivotal for its pro- or antitumor functions (26). Therefore, a study focusing on the regulation of $\beta$-catenin would highlight the pathological mechanism underlying PCa and also provide novel treatment targets. The results of the present study revealed that oldhamianoside II decreased the expression level of $\beta$-catenin and its downstream target 
genes, CCND1 and c-Jun, resulting in the suppression of $\mathrm{PCa}$ growth. Notably, oldhamianoside II was able to promote the proteasome-mediated degradation of $\beta$-catenin. These results further supported the potential application of oldhamianoside II in the treatment of PCa.

Wnt/ $\beta$-catenin signaling is frequently activated due to the suppression of its antagonists (27). To further investigate the mechanism underlying the effects of oldhamianoside II on Wnt $/ \beta$-catenin signaling, the present study also detected the expression levels of Wnt antagonists, including AXIN2, PPP22B, DKK1 and SFRP5 (28). Oldhamianoside II treatment was found to induce the expression of these genes. A previous study demonstrated that antagonists of the Wnt signaling pathway inhibited its activity in various ways; among these, DKK1 inhibited the interaction between the receptor and its ligand (29), while AXIN2 and PPP2CB could form a destruction complex to degrade $\beta$-catenin (30). Together with the present study, these findings suggest that oldhamianoside II may inhibit PCa progression via targeting $\beta$-catenin through various mechanisms.

In conclusion, the present study demonstrated a novel molecular mechanism, involving the reversal of EMT and inhibition of $\beta$-catenin signaling, through which the anticancer effects of oldhamianoside II are mediated. Oldhamianoside II may act as a chemopreventive agent to inhibit or delay the progression of $\mathrm{PCa}$ by promoting $\beta$-catenin degradation and inhibiting its transcriptional activity. The potential clinical application of oldhamianoside II in PCa treatment requires further study.

\section{Acknowledgements}

Not applicable.

\section{Funding}

This study was supported by the National Natural Science Foundation of China (grant nos. 81572544 and 81772760), The Key Research and Development Project of Shandong (grant no. 2016GSF201166), Scientific research fund of Jinan University (XKY1522), Supporting Fund For Teacher's research of Jining Medical University (JY2017FS001), The Shandong Taishan Scholarship (grant no. tsqn20161076) and The Innovation Project of Shandong Academy of Medical Sciences.

\section{Availability of data and materials}

All data generated or analyzed during this study are included in this published article.

\section{Authors' contributions}

SL and LW contributed to study conception and design. KL, XZ, JZ, TW, HD, FJ, DL, YC and YL contributed to the acquisition of data. KL, XZ, SL and LW contributed to the analysis and interpretation of data.

\section{Ethics approval and consent to participate}

Not applicable.

\section{Consent for publication}

All authors approved the final version to be published.

\section{Competing interests}

The authors declare that they have no competing interests.

\section{References}

1. Chen W, Zheng R, Baade PD, Zhang S, Zeng H, Bray F, Jemal A, Yu XQ and He J: Cancer statistics in China, 2015. CA Cancer J Clin 66: 115-132, 2016.

2. Heidenreich A, Aus G, Bolla M, Joniau S, Matveev VB, Schmid HP and Zattoni F; European Association of Urology: EAU guidelines on prostate cancer. Eur Urol 53: 68-80, 2008.

3. Gonzalez DM and Medici D: Signaling mechanisms of the epithelial-mesenchymal transition. Sci Signal 7: ra8, 2014.

4. Kong D, Banerjee S, Ahmad A, Li Y, Wang Z, Sethi S and Sarkar FH: Epithelial to mesenchymal transition is mechanistically linked with stem cell signatures in prostate cancer cells. PLoS One 5: e12445, 2010.

5. Nauseef JT and Henry MD: Epithelial-to-mesenchymal transition in prostate cancer: Paradigm or puzzle? Nat Rev Urol 8: 428-439, 2011.

6. Giles RH, van Es JH and Clevers H: Caught up in a Wnt storm: Wnt signaling in cancer. Biochim Biophys Acta 1653: 1-24, 2003.

7. Rabbani SA, Arakelian A and Farookhi R: LRP5 knockdown: Effect on prostate cancer invasion growth and skeletal metastasis in vitro and in vivo. Cancer Med 2: 625-635, 2013.

8. Dai J, Hall CL, Escara-Wilke J, Mizokami A, Keller JM and Keller ET: Prostate cancer induces bone metastasis through Wnt-induced bone morphogenetic protein-dependent and independent mechanisms. Cancer Res 68: 5785-5794, 2008.

9. Sun JY, Zhong Y, Yin JT, Liu L, Wang B and Zuo CX: Isolation and structure identification of triterpenoids from Gypsophila oldhamiana. Chin Trad Herbal Drugs 36: 644-646, 2005.

10. Wang FL, Sun JY, Wang Y, Mu YL, Liang YJ, Chong ZZ, Qin SH and Yao QQ: Oldhamianoside II, a new triterpenoidsaponin, prevents tumor growth via inducing cell apoptosis and inhibiting angiogenesis. Oncol Res 20: 369-376, 2013.

11. Wang L, Zhang J, Yang X, Chang YW, Qi M, Zhou Z, Zhang J and Han B: SOX4 is associated with poor prognosis in prostate cancer and promotes epithelial-mesenchymal transition in vitro. Prostate Cancer Prostatic Dis 16: 301-307, 2013.

12. Wang L, Zheng $\mathrm{Y}, \mathrm{Xu} \mathrm{H}$, Yan $\mathrm{X}$ and Chang X: Investigate pathogenic mechanism of TXNDC5 in rheumatoid arthritis. PLoS One 8: e53301, 2013.

13. Livak KJ and Schmittgen TD: Analysis of relative gene expression data using real-time quantitative PCR and the 2(-Delta Delta C(T)) method. Methods 25: 402-408, 2001.

14. Wang L, Li Y, Yang X, Yuan H, Li X, Qi M, Chang YW, Wang C, $\mathrm{Fu} \mathrm{W}$, Yang M, et al: ERG-SOX4 interaction promotes epithelial-mesenchymal transition in prostate cancer cells. Prostate 74: 647-658, 2014.

15. Smith BN and Odero-Marah VA: The role of Snail in prostate cancer. Cell Adh Migr 6: 433-441, 2012.

16. MacDonald BT, Tamai K and He X: Wnt/beta-catenin signaling: Components, mechanisms, and diseases. Dev Cell 17: 29-26, 2009.

17. Logan CY and Nusse R: The Wnt signaling pathway in development and disease. Annu Rev Cell Dev Biol 20: 781-810, 2004.

18. Fischer JA: Deubiquitinating enzymes: Their roles in development, differentiation, and disease. Int Rev Cytol 229: 43-72, 2003.

19. Arend RC,Londoño-Joshi AI, Straughn JM Jr and Buchsbaum DJ: The Wnt/ $\beta$-catenin pathway in ovarian cancer: A review. Gynecol Oncol 131: 772-779, 2013.

20. Koch A, Waha A, Hartmann W, Hrychyk A, Schüller U, Waha A Wharton KA Jr, Fuchs SY, von Schweinitz D and Pietsch T: Elevated expression of Wnt antagonists is a common event in hepatoblastomas. Clin Cancer Res 11: 4295-4304, 2005.

21. Thiery JP, Acloque H, Huang RY and Nieto MA: Epithelial-mesenchymal transitions in development and disease. Cell 139: 871-890, 2009.

22. Bullions LC and Levine AJ: The role of beta-cateninin cell adhesion, signal transduction, and cancer. Curr Opin Oncol 10: 81-87, 1998. 
23. Mulholland DJ, Dedhar S, Coetzee GA and Nelson CC: Interaction of nuclear receptors with the Wnt/beta-catenin/Tcf signaling axis: Wnt you like to know? Endocr Rev 26: 898-915, 2005.

24. Sundram V, Chauhan SC, Ebeling M and Jaggi M: Curcumin attenuates $\beta$-catenin signaling in prostate cancer cells through activation of protein kinase D1. PLoS One 7: e35368, 2012.

25. Yokoyama NN, Shao S, Hoang BH, Mercola D and Zi X: Wnt signaling in castration-resistant prostate cancer: Implications for therapy. Am J Clin Exp Urol 15: 27-44, 2014

26. Park JH, Kwon HY, Sohn EJ, Kim KA, Kim B, Jeong SJ, Song JH, Koo JS and $\mathrm{Kim} \mathrm{SH}$ : Inhibition of $\mathrm{Wnt} / \beta$-catenin signaling mediates ursolic acid-induced apoptosis in $\mathrm{PC}-3$ prostate cancer cells. Pharmacol Rep 65: 1366-1374, 2013.
27. Robinson DR, Zylstra CR and Williams BO: Wnt signaling and prostate cancer. Curr Drug Targets 9: 571-580, 2008.

28. Kypta RM and Waxman J: Wnt/ $\beta$-catenin signaling in prostate cancer. Nat Rev Urol 9: 418-428, 2012.

29. Kagey $\mathrm{MH}$ and He X: Rationale for targeting the Wnt signalling modulator Dickkopf-1 for oncology. Br J Pharmacol 174: 4637-4650, 2017

30. Cheng AS, Lau SS, Chen Y, Kondo Y, Li MS, Feng H, Ching AK, Cheung KF, Wong HK, Tong JH, et al: EZH2-mediated concordant repression of Wnt antagonists promotes $\beta$-catenin-dependent hepatocarcinogenesis. Cancer Res 71: 4028-4039, 2011. 